\title{
Mecanismos fisiológicos em híbridos de citros sob estresse salino em cultivo hidropônico ${ }^{1}$
}

\author{
Luderlândio de A. Silva², Marcos E. B. Brito 3 , Francisco V. da S. Sá, \\ Rômulo C. L. Moreira ${ }^{5}$, Walter dos S. Soares Filho ${ }^{6} \&$ Pedro D. Fernandes ${ }^{7}$ \\ ${ }^{1}$ Trabalho selecionado do II INOVAGRI International Meeting, realizado de 13 a 16 de abril de 2014, Fortaleza - CE, Brasil \\ ${ }^{2}$ UAGRA/CCTA/UFCG. Pombal, PB. E-mail: luderlandioandrade@gmail.com \\ ${ }^{3}$ UAGRA/CCTA/UFCG. Pombal, PB. E-mail: marcoseric@ccta.ufcg.edu.br (Autor correspondente) \\ ${ }^{4}$ UAGRA/CCTA/UFCG. Pombal, PB. E-mail: vanies_agronomia@hotmail.com \\ ${ }^{5}$ UAGRA/CCTA/UFCG. Pombal, PB. E-mail: romulocarantino@gmail.com \\ ${ }^{6}$ Embrapa Mandioca e Fruticultura. Cruz das Almas, BA. E-mail: walter.soares@embrapa.br \\ ${ }^{7}$ UAEA/CTRN/UFCG. Campina Grande, PB. E-mail: pdantas@pq.cnpq.br
}

\section{Palavras-chave:}

Citrus spp.

Poncirus

solução de Hoagland

trocas gasosas

\section{R E S U M O}

Objetivou-se estudar os mecanismos fisiológicos de percepção e tolerância ao estresse salino de híbridos de citros em cultivo hidropônico. O experimento foi realizado em ambiente protegido do Centro de Ciências e Tecnologia Agroalimentar - CCTA, da Universidade Federal de Campina Grande - UFCG, Pombal, PB. Foram avaliados, em blocos casualizados, em esquema fatorial (2x5), dois níveis de salinidade da água de irrigação $\left(0,3\right.$ e 4,0 dS $\left.\mathrm{m}^{-1}\right)$ e cinco híbridos recomendados para uso como porta-enxerto [HTR - 069, HTR - 116, HTR - 127, LCR x TR - 001 e TSKC x (LCR x TR) - 040)], com três repetições. Os genótipos foram cultivados em sistema hidropônico adaptado em vasos de Leonard, utilizando solução de Hoagland. O início da aplicação da solução nutritiva com uso de águas salinas ocorreu aos 90 dias após a semeadura, quando se procedeu as avaliações quanto às trocas gasosas e fluorescência do PSII às 0,24 e 48 horas após aplicação dos tratamentos a fim de determinar o efeito do estresse salino sobre os atributos fisiológicos. A fluorescência inicial, a condutância estomática e a eficiência no uso da água foram as variáveis que mais permitem evidenciar a percepção do estresse neste trabalho. O híbrido TSKC x (LCR x TR) - 040 foi o mais tolerante à salinidade, seguido do híbrido HTR - 069. O híbrido LCR x TR - 001 foi o mais sensível à salinidade, por ter sua atividade do PSII comprometida.
Key words:

Citrus spp.

Poncirus

Hoagland solution

gas exchange

\section{Physiological mechanisms in citrus hybrids under saline stress in hydroponic system}

\begin{abstract}
A B S T R A C T
In hydroponic system the physiological mechanisms of perception and tolerance to salt stress of citrus hybrids was studied. The experiment was carried out in greenhouse at the Center of Agrifood Science and Technology - CCTA, Federal University of Campina Grande, Pombal, PB, Brazil. A randomized block design was used in a factorial scheme (2x5), with two water salinity levels of solution $\left(0.3\right.$ and $\left.4.0 \mathrm{dS} \mathrm{m}^{-1}\right)$ and five hybrids with potential for use as citrus rootstocks [HTR - 069, HTR - 116, HTR - 127, LCR x TR - 001 and TSKC x (LCR x TR) - 040)], with three replications. The hybrids were grown in hydroponic system, using Leonard's pots and Hoagland's solution. The application of salinized solution was started at 90 days after sowing, when the gas exchange and PSII fluorescence were evaluated at 0,24 and 48 hours after imposition of treatments, in order to determine the time interval for beginning of salt stress by physiological aspects. The initial fluorescence, stomatal conductance and water use efficiency were the variables that best described the perception of stress in this study. The hybrid TSKC $\mathrm{x}$ (LCR $\mathrm{x}$ TR) - 040 proved to be more tolerant to salinity, followed by hybrid HTR - 069. The hybrid LCR x TR - 001 was the most sensitive to salinity, due to damages in its PSII activity.
\end{abstract}

\section{INTRODUÇÃo}

O agronegócio dos citros é uma das mais importantes atividades do cenário agrícola brasileiro, tanto sob o ponto de vista econômico quanto social, sendo o cultivo da laranjeira doce [Citrus sinensis (L.) Osbeck], especialmente a 'Pera', o mais representativo, com uma participação de, aproximadamente,
90\% da citricultura brasileira, destacando-se no grupo dos limoeiros verdadeiros - limeiras ácidas a cultivar Tahiti ( $C$. latifolia (Yu. Tanaka) Tanaka) (Agrianual, 2010). Dentre as variedades porta-enxerto, a mais explorada no país é o limoeiro 'Cravo' (C. limonia Osbeck) (Mattos Junior et al., 2005).

A citricultura vem se expandido no Nordeste do Brasil, no entanto, com baixos níveis de produtividade, em razão, 
principalmente, da escassez de água ao longo do ano, notadamente em períodos que coincidem com temperaturas elevadas, entretanto esse problema pode ser contornado mediante a adoção de técnicas como a irrigação (Fernandes et al., 2011; Silva et al., 2012).

A região Nordeste, ainda, é caracterizada por possuir áreas com clima semiárido, onde os problemas de indisponibilidade hídrica são agravados tanto por escassez quantitativa de água, decorrente da superioridade dos valores de evapotranspiração em relação aos de precipitação, quanto qualitativa, em virtude de altas concentrações de sais na água de poços, açudes e rios (Medeiros et al., 2003).

Diante do problema da presença de sais nas águas de irrigação, o que vem a ocasionar aumento da concentração de sais no solo, deve-se procurar alternativas ao cultivo, a exemplo do uso de genótipos que sejam tolerantes a salinidade, já que, segundo Ayers \& Westcot (1999), Brito et al. (2008) e Fernandes et al. (2011), a tolerancia a salinidade é variável entre espécies, entre genótipos e entre fases de desenvolvimento da cultura.

Nesse contexto, a identificação de genótipos com diversidade de respostas às estresse abiótico, como o salino, é interessante dentro de programas de melhoramento genético, sendo importante conhecer mecanismos relacionados a tais respostas diferenciais (Nascimento et al., 2012).

Acrescenta-se que, no Brasil, o uso intensivo do limoeiro 'Cravo', apesar de sua expressiva tolerância à seca, além de outros atributos de valor agronômico, não permite atingir o máximo potencial produtivo de todas as variedades copa, além de deixar o sistema produtivo de citros vulnerável a problemas bióticos e abióticos (Matos Junior, 2005), o que indica a necessidade de se promover um programa de diversificação de cultivares porta-enxerto, sendo uma das características importantes a tolerância a salinidade.

$\mathrm{Na}$ avaliação das diferentes respostas de variedades de importância agrícola, como a beterraba e eucalipto e citros, aos efeitos da salinidade, estudos relacionados à fotossíntese e à fluorescência da clorofila têm se mostrado bem sucedidos (Jamil et al., 2007; Mendonça et al., 2010; Brito et al., 2012), acreditando-se ser possível identificar, na fase inicial de crescimento de porta-enxertos de citros, se determinado genótipo apresenta tolerância e/ou desenvolve mecanismos fisiológicos para percepção do estresse salino. De acordo com Flowers \& Flowers (2005), Brito et al. (2008) e Fernandes et al. (2011), as respostas variam entre espécies, bem como dentro de uma mesma espécie, nos diferentes estádios de desenvolvimento da planta.

Assim sendo, objetivou-se estudar os mecanismos fisiológicos de percepção e tolerância ao estresse salino em híbridos de citros, com finalidade de uso como porta-enxerto, em cultivo hidropônico.

\section{Material e Métodos}

$\mathrm{O}$ experimento foi desenvolvido em ambiente protegido, no Centro de Ciências e Tecnologia Agroalimentar (CCTA) da Universidade Federal de Campina Grande (UFCG), município de Pombal - PB, nas coordenadas geográficas $6^{\circ} 47^{\prime} 20^{\prime \prime}$ de latitude $\mathrm{S}$ e $37^{\circ} 48^{\prime} 01^{\prime \prime}$ de longitude $\mathrm{W}$, a uma altitude média de $184 \mathrm{~m}$.

Foram estudados em blocos casualizados, utilizando esquema fatorial $2 \times 5$, dois níveis de salinidade da água usada para preparar as soluções nutritivas $\left(\mathrm{S}_{1}=0,3\right.$ e $\left.\mathrm{S}_{2}=4,0 \mathrm{dS} \mathrm{m}^{-1}\right)$ e cinco híbridos de citros com potencial de uso como portaenxertos [híbrido trifoliado (HTR) - 069, HTR - 116, HTR - 127, limoeiro 'Cravo' (LCR) x Poncirus trifoliata (L.) Raf. (TR) - 001 e tangerineira 'Sunki' comum C. sunki (Hayata) hort. ex Tanaka (TSKC) x (LCR x TR) - 040]. Foram constituídos três blocos e quatro plantas úteis por parcela, dentro de cada repetição, em cada nível de salinidade. Os mencionados híbridos foram obtidos pelo Programa de Melhoramento Genético de Citros da Embrapa Mandioca e Fruticultura - PMG Citros.

Os níveis de salinidade da água testados foram estabelecidos em razão a salinidade limiar $\left(2 \mathrm{dS} \mathrm{m}^{-1}\right)$ sendo um nível abaixo e outro acima da salinidade limiar de variedades de citros, descrita por Singh et al. (2003).

A semeadura e o crescimento das plantas dos distintos híbridos ocorreram em recipientes com capacidade de 1.500 $\mathrm{mL}$ de substrato, tendo como substrato fibra de coco lavada, de modo a garantir que não houvesse interferência de sais presentes no material, assim como disponibilidade de nutrientes, por se tratar de um substrato inerte. Ressalta-se que as soluções nutritivas usadas seguiram as recomendações de Hoagland \& Arnon (1950), sendo, porém, acrescentado 25\% de ferro EDTA, a fim de suprir as necessidades nutricionais dos genótipos (Tabela 1) observado em ensaio preliminar.

As sementes, devidamente selecionadas e tratadas com fungicida disulfato de thiram ( $4 \mathrm{~g} \mathrm{~kg}^{-1}$ de sementes), foram semeadas na razão de três por recipiente, sendo cobertas com o substrato. Os recipientes usados para cultivo das plantas de citros foram os vasos de Leonard adaptados com garrafas Pet (Santos et al., 2009). A parte superior dos vasos foi preenchida com 1,5 L do substrato (pó de coco) e, na parte inferior, permaneceu a solução nutritiva de Hoagland (Hoagland \& Arnon, 1950) sob fluxo contínuo.

Após o preparo dos vasos de Leonard, os mesmos foram envolvidos com plástico dupla face, visando reduzir o processo de evaporação da solução, a qual foi verificada diariamente e, quando necessário, completada conforme tratamento, garantindo que o substrato ficasse com umidade sempre próxima à capacidade de campo.

Após a germinação das sementes, quando os seedlings (pésfrancos ou plantas oriundas de sementes) apresentavam três ou

Tabela 1. Concentração dos nutrientes na solução nutritiva para cultivo hidropônico de citros ${ }^{\#}$

\begin{tabular}{|c|c|c|c|c|c|c|c|c|c|c|c|c|}
\hline \multirow{2}{*}{ Nutrientes } & $\mathbf{N}$ & $P$ & K & $\mathrm{Ca}$ & $\mathrm{Mg}$ & $S$ & $\mathrm{Fe}$ & Mn & B & $\mathrm{Cu}$ & $\mathrm{Zn}$ & Mo \\
\hline & \multicolumn{12}{|c|}{$\left(\mathrm{mmol} \mathrm{L}^{-1}\right)$} \\
\hline Concentração & 15 & 1 & 6 & 5 & 2 & 2 & 0,0625 & 0,01 & 0,05 & 0,003 & 0,0008 & 0,001 \\
\hline
\end{tabular}

\# Adaptado de Hoagland \& Arnon (1950) 
mais pares de folhas verdadeiras, deixou-se desenvolver apenas um indivíduo por recipiente, tomando-se o cuidado de manter somente aqueles que representassem o padrão de planta de cada genótipo, no sentido de selecionar seedlings de origem nucelar, representantes, portanto, de suas respectivas plantas-mãe. Adotaram-se todos os cuidados de controle de ervas-daninhas e de prevenção e controle de pragas, recomendados na produção de mudas cítricas (Mattos Júnior et al., 2005).

As águas para a mistura com as soluções nutritivas foram preparadas de modo a se ter uma proporção equivalente de $7 \mathrm{Na}: 2 \mathrm{Ca}: 1 \mathrm{Mg}$, utilizando os sais $\mathrm{NaCl}, \mathrm{CaCl}_{2} \cdot 2 \mathrm{H}_{2} \mathrm{O}$ e $\mathrm{MgCl}_{2} \cdot 6 \mathrm{H}_{2} \mathrm{O}$, respectivamente, relação esta que reflete os íons predominantes em fontes de água utilizada para irrigação, em pequenas propriedades do Nordeste brasileiro (Medeiros et al., 2003). O preparo das soluções com as diferentes condutividades elétricas (CE) foi por adição dos sais a água até ser atingido o nível desejado de $\mathrm{CE}$, conferindo-se os valores com um condutivímetro portátil ajustado para a temperatura de $25^{\circ} \mathrm{C}$.

A solução nutritiva foi preparada com água destilada e possuía uma condutividade elétrica de $2,3 \mathrm{dS} \mathrm{m}^{-1}$, esta, então, foi misturada às águas com $0,3 \mathrm{dS} \mathrm{m}^{-1}(\mathrm{~S} 1)$ e $4,3 \mathrm{dS} \mathrm{m}^{-1}\left(\mathrm{~S}_{2}\right)$, sendo assim, as soluções disponíveis às plantas possuíam 2,6 e $6,3 \mathrm{dS} \mathrm{m}^{-1}$, respectivamente.

Aos 90 dias após a semeadura os híbridos de citros recomendados como porta-enxertos foram avaliados quanto ao estabelecimento fisiológico do estresse salino, mensurando-se a taxa de assimilação de $\mathrm{CO}_{2}(\mathrm{~A})\left(\mu \mathrm{mol} \mathrm{m} \mathrm{m}^{-2} \mathrm{~s}^{-1}\right)$, transpiração (E) ( $\mathrm{mmol} \mathrm{de}_{2} \mathrm{O} \mathrm{m}^{-2} \mathrm{~s}^{-1}$ ), condutância estomática (gs) (mol de $\left.\mathrm{H}_{2} \mathrm{Om}^{-2} \mathrm{~s}^{-1}\right)$ e a concentração interna de $\mathrm{CO}_{2}(\mathrm{Ci})$ na primeira folha madura contada a partir do ápice. Com esses dados quantificou-se a eficiência no uso da água (EUA) $(\mathrm{A} / \mathrm{T})[(\mu \mathrm{mol}$ $\left.\left.\mathrm{m}^{-2} \mathrm{~s}^{-1}\right)\left(\mathrm{mmol} \mathrm{H}_{2} \mathrm{O} \mathrm{m}^{-2} \mathrm{~s}^{-1}\right)^{-1}\right]$ e a eficiência instantânea da carboxilação Фc (A/Ci) (Konrad et al., 2005; Brito et al., 2012), utilizando o equipamento portátil de medição de fotossíntese "LCPro+" da ADC BioScientific Ltda. Nessas mesmas folhas, onde foram analisadas as trocas gasosas, foram colocadas pinças foliares e, após um período de $30 \mathrm{~min}$. de adaptação ao escuro (Konrad et al., 2005), ocasioão em que se determinou: a fluorescência inicial (Fo), a fluorescência máxima (Fm), a fluorescência variável (Fm-Fo) e a eficiência quântica do fotossistema II (Fv/Fm) (Maxwell \& Johnson, 2000) às 0, 24 e 48 horas após início da aplicação das soluções nutritivas adicionadas de água salina, usando-se o equipamento PEA Hansatech.

Os resultados obtidos foram avaliados mediante análise de variância pelo teste 'F'. Nos casos de significância, foi realizado o teste de agrupamento de médias (Scott $\&$ Knott até 0,05 de probabilidade) para o fator porta-enxerto em cada nível de salinidade estudado (Ferreira, 2000).

\section{Resultados e Discussão}

Não foram constatadas diferenças significativas entre os híbridos quanto às trocas gasosas antes da aplicação dos tratamentos (Tabela 2). Entretanto, após as primeiras 24 horas da aplicação das soluções com os níveis de salinidade, observou-se interações significativas entre os fatores níveis de salinidade da água e híbridos de citros em todas as variáveis de trocas gasosas, à exceção da concentração interna de $\mathrm{CO}_{2}$, para a qual foram observadas apenas diferenças significativas $(p<0,05)$ entre os híbridos de citros.

Após 48 horas do início da aplicação dos tratamentos, o efeito da interação entre os fatores estudados foi significativo, apenas, para a taxa de assimilação de $\mathrm{CO}_{2}$. Contudo, em relação à variável condutância estomática foram observados efeitos dos dois fatores isolados (Tabela 2). No tocante à concentração

Tabela 2. Resumo da análise de variância para a concentração interna de $\mathrm{CO}_{2}(\mathrm{Ci})$, transpiração (E), condutância estomática (gs), taxa de assimilação de $\mathrm{CO}_{2}(\mathrm{~A})$, eficiência no uso da água (EUA) e eficiência instantânea da carboxilação (Фc), referentes aos híbridos de citros às 0,24 e 48 h após início da aplicação de solução nutritiva salinizada em cultivo hidropônico

\begin{tabular}{|c|c|c|c|c|c|c|c|}
\hline \multirow{2}{*}{ Fontes de variação } & \multirow{2}{*}{ GL } & \multicolumn{6}{|c|}{ Quadrado médio } \\
\hline & & Ci Oh & E Oh & gs $\mathrm{Oh}$ & $\mathrm{AOh}$ & EUA Oh & $\Phi c$ Oh \\
\hline Híbridos & 4 & $196,44^{\mathrm{NS}}$ & $0,24^{\mathrm{NS}}$ & $0,00115^{\mathrm{NS}}$ & $2,41^{\mathrm{NS}}$ & $0,10^{\mathrm{NS}}$ & $0,00003^{N S}$ \\
\hline Blocos & 2 & $222,61^{\mathrm{NS}}$ & $0,25^{\mathrm{NS}}$ & 0,00008 NS & $0,04^{\mathrm{NS}}$ & $0,58^{*}$ & $1,81 \mathrm{E}^{-0008 \mathrm{NS}}$ \\
\hline Erro & 8 & 111,86 & 0,12 & 0,00063 & 1,17 & 0,06 & 0,000017 \\
\hline \multirow[t]{2}{*}{$\mathrm{CV}$} & & 4,01 & 18,35 & 28,43 & 23,14 & 10,38 & 22,77 \\
\hline & & Ci 24h & E 24h & Gs 24h & A 24h & EUA 24h & Фc 24h \\
\hline Salinidade & 1 & $392,40^{\mathrm{NS}}$ & $0,76^{* *}$ & $0,0029 * *$ & $10,65^{\star \star}$ & $0,46^{\mathrm{NS}}$ & $0,00015^{\star}$ \\
\hline Híbridos & 4 & $1.693,66^{\star}$ & $1,68^{* *}$ & 0,0064 ** & $26,33^{\star *}$ & $1,45^{\star *}$ & $0,00047 * *$ \\
\hline Salinidade x Híbridos & 4 & $601,86^{\text {NS }}$ & $0,41 * *$ & $0,0013^{\star}$ & $4,48^{\star}$ & $0,60^{*}$ & $0,00008^{*}$ \\
\hline Blocos & 2 & $612,50^{\mathrm{NS}}$ & 0,26 NS & $0,0002^{N S}$ & $0,85^{\mathrm{NS}}$ & $0,06^{\mathrm{NS}}$ & $0,00002^{\mathrm{NS}}$ \\
\hline Erro & 18 & 217,30 & 0,08 & 0,0003 & 1,28 & 0,15 & 0,00002 \\
\hline \multirow[t]{2}{*}{ CV } & & 5,55 & 18,01 & 21,19 & 24,05 & 14,06 & 26,77 \\
\hline & & Ci $48 \mathrm{~h}$ & E 48h & gs $48 \mathrm{~h}$ & A 48h & EUA 48h & $\Phi c 48 \mathrm{~h}$ \\
\hline Salinidade & 1 & $307,20^{\text {NS }}$ & $0,20^{N S}$ & $0,0029 *$ & $1,92^{\mathrm{NS}}$ & $0,06^{\mathrm{NS}}$ & $0,000006^{\mathrm{NS}}$ \\
\hline Híbridos & 4 & $1.866,45^{\star *}$ & $1,35^{* *}$ & $0,0074^{\star *}$ & 25,16 ** & $1,54^{\star *}$ & 0,000450 ** \\
\hline Salinidade x Híbridos & 4 & $512,28^{\text {NS }}$ & $0,03^{\text {NS }}$ & $0,0011^{\mathrm{NS}}$ & $2,12^{\star}$ & $0,49^{\mathrm{NS}}$ & $0,000037^{\text {NS }}$ \\
\hline Blocos & 2 & $1.534,30$ & $0,14^{\mathrm{NS}}$ & $0,0001^{\mathrm{NS}}$ & $0,57^{\mathrm{NS}}$ & $0,11^{\mathrm{NS}}$ & $0,000034^{\mathrm{NS}}$ \\
\hline Erro & 18 & 357,00 & 0,08 & 0,0004 & 0,60 & 0,20 & 0,000015 \\
\hline $\mathrm{CV}$ & & 7,15 & 17,95 & 24,05 & 15,05 & 14,07 & 19,34 \\
\hline
\end{tabular}

\footnotetext{
* ${ }^{\star *}=$ significante ao nível de 0,05 e 0,01 de probabilidade; ${ }^{N S}=$ não significativo; $\mathrm{GL}=$ grau de liberdade; CV= coeficiente de variação.
} 
interna de $\mathrm{CO}_{2}$, transpiração, eficiência do uso da água e eficiência instantânea da carboxilação foram observadas apenas diferenças entre os híbridos ( $<<0,01)$ (Tabela 2).

Nos híbridos HTR-127, LCR x TR - 001 e HTR-116 constatou-se as maiores concentrações internas de $\mathrm{CO}_{2}$ (Tabela 3 ), fato este que pode indicar baixa atividade da enzima ribulose-1,5-bisfosfato carboxilase-oxigenase (rubisco), assim como identificado por Machado et al. (2005) ao estudar três variedades copa de citros sob diferentes condições ambientais, e por Konrad et al. (2005) estudando cultivares de café sob estresse por alumínio, os quais relacionam essa resposta a uma baixa atividade dos estômatos, já que estes são responsáveis por regular os processos de trocas gasosas (Taiz \& Zaiger, 2009). Tal situação, ainda, pode ser atribuída a algum fator não estomático, como a falta de ATP e de NADPH provenientes da cadeia transportadora de elétrons do fotossistema II, o que poderá ser confirmado quando se estudar a eficiência instantânea da carboxilação e a fluorescência da clorofila.

De acordo com Hussain et al. (2012), os efeitos primários da salinidade citros referem-se à diminuição da condutância estomática, levando à redução da difusão de $\mathrm{CO}_{2}$ para a planta e, consequentemente, da taxa de fotossíntese líquida, o que é confirmado por Magalhães Filho et al. (2008), relatando que o fechamento dos estômatos ocorre devido à desidratação das células-guarda, ou por resposta hormonal, conforme foi observado nesses genótipos.

Quanto à transpiração, após 24 horas de submissão as soluções salinizadas, verificou-se efeito significativo do aumento da concentração da solução apenas no genótipo HTR-127, ocorrendo redução nos valores, implicando na percepção do estresse salino nesse momento, havendo estabilização na avaliação às 48 horas (Tabela 3 ), destacando-se que neste genótipo, a maior média entre os materiais quando submetido a solução preparada com água de $0,3 \mathrm{dS} \mathrm{m}^{-1}$, e a menor média quando exposto a solução preparada com água de $4,0 \mathrm{dS}$ $\mathrm{m}^{-1}$. Além disso, o HTR-069 também sofreu reduções em sua transpiração sob condições de salinidade, resposta esta, todavia, somente observada na avaliação às 48 horas, ademais, acrescenta-se que as maiores médias relativas a transpiração são observadas nesse genótipo, tanto sob solução de $0,3 \mathrm{dS} \mathrm{m}^{-1}$ quanto de $4,0 \mathrm{dS} \mathrm{m}^{-1}$ as $24 \mathrm{e} 48$ horas de exposição, possibilitando visualizar o potencial fisiológico do genótipo, assim como identificado por Brito et al. (2012), estudando este material sob condições de estresse hídrico e enxertado com o pomeleiro 'Star Ruby' e limeira ácida 'Tahiti'.

Deve-se salientar que, tanto no híbrido trifoliado HTR-127 quanto no HTR-069, de maneira semelhante ao que ocorreu na transpiração, notou-se redução na condutância estomática (Tabela 3), deduzindo-se que ocorreu estresse, pois a redução na condutância estomática é tida como um mecanismo de tolerância à salinidade que reduz o processo transpiratório, como também regula a entrada de água nas plantas, com isso, evitando a entrada de sais e, dessa forma, a toxicidade por íons específicos (Flowers \& Flowers, 2005), diante disso pode-se dizer que a condutância estomática é uma variável importante
Tabela 3. Teste de médias referente à concentração interna de $\mathrm{CO}_{2}$, transpiração, condutância estomática, fotossíntese, eficiência no uso da água e à eficiência instantânea da carboxilação (Фc) de híbridos de citros submetidos à salinidade da água sob cultivo hidropônico, durante 0,24 e 48 horas

\begin{tabular}{|c|c|c|c|c|c|}
\hline \multirow{3}{*}{ Híbridos } & \multirow{2}{*}{$\begin{array}{c}\text { O hora } \\
\mathbf{0 , 3} \\
\end{array}$} & \multicolumn{2}{|c|}{24 horas } & \multicolumn{2}{|c|}{48 horas } \\
\hline & & 0,3 & 4,0 & 0,3 & 4,0 \\
\hline & \multicolumn{5}{|c|}{$\mathrm{dS} \mathrm{\textrm {m } ^ { - 1 }}$} \\
\hline \multicolumn{6}{|c|}{ Concentração interna de $\mathrm{CO}_{2}(\mathrm{Ci})\left(\mathrm{mmol} \mathrm{mol}^{-1}\right)$} \\
\hline HTR - 127 & $274 \mathrm{a}$ & $265 \mathrm{aA}$ & $290 \mathrm{aA}$ & $284 \mathrm{aA}$ & $266 \mathrm{aA}$ \\
\hline LCR x TR - 001 & $251 \mathrm{a}$ & $254 a A$ & $282 \mathrm{aA}$ & $258 \mathrm{aA}$ & $267 a A$ \\
\hline TSKC $\times($ LCR $\times$ TR $)-040$ & $264 \mathrm{a}$ & $255 \mathrm{bA}$ & $234 \mathrm{bA}$ & $246 \mathrm{bA}$ & $235 \mathrm{bA}$ \\
\hline HTR - 116 & $266 \mathrm{a}$ & $284 a A$ & $286 \mathrm{aA}$ & $278 \mathrm{aA}$ & $294 a A$ \\
\hline HTR - 069 & $263 \mathrm{a}$ & $251 \mathrm{bA}$ & $253 \mathrm{bA}$ & $270 \mathrm{bA}$ & $242 \mathrm{bA}$ \\
\hline \multicolumn{6}{|c|}{ Transpiração (E) $\left(\mathrm{mmol}\right.$ de $\left.\mathrm{H}_{2} \mathrm{O} \mathrm{m}^{-2} \mathrm{~s}^{-1}\right)$} \\
\hline HTR - 127 & $2,12 \mathrm{a}$ & $2,49 \mathrm{aA}$ & $1,35 \mathrm{cB}$ & $1,94 \mathrm{aA}$ & $1,99 a A$ \\
\hline LCR $x$ TR - 001 & $2,00 \mathrm{a}$ & $0,93 \mathrm{bA}$ & $1,08 \mathrm{cA}$ & $1,25 \mathrm{bA}$ & $1,07 \mathrm{cA}$ \\
\hline TSKC $\times($ LCR $\times$ TR $)-040$ & $1,90 \mathrm{a}$ & $2,04 \mathrm{aA}$ & $1,69 \mathrm{bA}$ & $1,88 \mathrm{aA}$ & $1,65 \mathrm{bA}$ \\
\hline HTR - 116 & $1,40 \mathrm{a}$ & $1,29 \mathrm{bA}$ & $0,92 \mathrm{cA}$ & $1,03 \mathrm{bA}$ & $0,94 \mathrm{cA}$ \\
\hline HTR - 069 & $2,04 \mathrm{a}$ & $2,13 \mathrm{aA}$ & $2,25 \mathrm{aA}$ & $2,20 \mathrm{aA}$ & $1,84 \mathrm{aB}$ \\
\hline \multicolumn{6}{|c|}{ Condutância estomática (gs) (mol de $\left.\mathrm{H}_{2} \mathrm{O} \mathrm{m}^{-2} \mathrm{~s}^{-1}\right)$} \\
\hline HTR - 127 & $0,10 \mathrm{a}$ & $0,14 \mathrm{aA}$ & $0,07 b B$ & $0,12 \mathrm{aA}$ & $0,13 a A$ \\
\hline LCR $x$ TR -001 & $0,08 \mathrm{a}$ & $0,04 \mathrm{bA}$ & $0,05 \mathrm{bA}$ & $0,07 \mathrm{bA}$ & $0,05 \mathrm{bA}$ \\
\hline TSKC $\times($ LCR $\times$ TR $)-040$ & $0,09 \mathrm{a}$ & $0,10 \mathrm{aA}$ & $0,09 \mathrm{aA}$ & $0,11 \mathrm{aA}$ & $0,10 \mathrm{aA}$ \\
\hline HTR - 116 & $0,05 \mathrm{a}$ & $0,06 \mathrm{bA}$ & $0,04 \mathrm{bA}$ & $0,05 \mathrm{bA}$ & $0,04 \mathrm{bA}$ \\
\hline HTR - 069 & $0,10 \mathrm{a}$ & $0,12 \mathrm{aA}$ & $0,12 \mathrm{aA}$ & $0,16 \mathrm{aA}$ & $0,09 \mathrm{aB}$ \\
\hline \multicolumn{6}{|c|}{ Taxa de assimilação de $\mathrm{CO}_{2}(\mathrm{~A})\left(\mu \mathrm{mol} \mathrm{m} \mathrm{m}^{-2} \mathrm{~s}^{-1}\right)$} \\
\hline HTR - 127 & $4,92 \mathrm{a}$ & $7,13 \mathrm{aA}$ & $2,95 b B$ & $5,44 \mathrm{bB}$ & $6,86 \mathrm{aA}$ \\
\hline LCR x TR - 001 & $5,11 \mathrm{a}$ & $2,80 \mathrm{bA}$ & $2,40 \mathrm{bA}$ & $4,23 c A$ & $2,99 \mathrm{bA}$ \\
\hline TSKC $\times($ LCR $\times$ TR $)-040$ & $4,85 \mathrm{a}$ & $6,17 \mathrm{aA}$ & $6,42 \mathrm{aA}$ & $6,85 \mathrm{aA}$ & $6,54 \mathrm{aA}$ \\
\hline HTR - 116 & $3,13 \mathrm{a}$ & $2,98 \mathrm{bA}$ & $2,04 \mathrm{bA}$ & $2,76 \mathrm{dA}$ & $2,00 \mathrm{bA}$ \\
\hline HTR - 069 & $5,43 \mathrm{a}$ & $7,44 \mathrm{aA}$ & $6,74 \mathrm{bA}$ & $7,81 \mathrm{aA}$ & $6,16 \mathrm{aB}$ \\
\hline \multicolumn{6}{|c|}{ Eficiência no uso da água (EUA) $(A / T)\left[\left(\mu \mathrm{mol} \mathrm{m}^{-2} \mathrm{~s}^{-1}\right)\left(\mathrm{mol} \mathrm{H}_{2} \mathrm{O} \mathrm{m}^{-2} \mathrm{~s}^{-1}\right)^{-1}\right]$} \\
\hline HTR - 127 & $2,31 \mathrm{a}$ & $2,81 \mathrm{bA}$ & $2,14 \mathrm{bA}$ & $2,80 \mathrm{aA}$ & $3,43 \mathrm{bA}$ \\
\hline LCR $x$ TR - 001 & $2,66 \mathrm{a}$ & $3,01 b A$ & $2,32 b B$ & $3,42 \mathrm{aA}$ & $2,80 \mathrm{bB}$ \\
\hline TSKC $\times($ LCR $\times$ TR $)-040$ & $2,61 \mathrm{a}$ & $3,01 b B$ & $3,82 \mathrm{aA}$ & $3,67 a \mathrm{~B}$ & $4,05 \mathrm{aA}$ \\
\hline HTR - 116 & $2,32 \mathrm{a}$ & $2,31 b A$ & $2,21 b A$ & $2,80 \mathrm{aA}$ & $2,18 \mathrm{bA}$ \\
\hline HTR - 069 & $2,67 \mathrm{a}$ & $3,52 \mathrm{aA}$ & $2,94 \mathrm{bA}$ & $3,60 \mathrm{aA}$ & $3,36 \mathrm{bA}$ \\
\hline \multicolumn{6}{|c|}{ Eficiência instantânea da carboxilação - Фc (A/Ci) } \\
\hline HTR - 127 & $0,018 \mathrm{a}$ & $0,027 \mathrm{aA}$ & $0,010 \mathrm{bB}$ & $0,019 \mathrm{bB}$ & $0,026 \mathrm{aA}$ \\
\hline LCR x TR - 001 & $0,020 \mathrm{a}$ & $0,011 \mathrm{bA}$ & $0,009 \mathrm{bA}$ & $0,016 \mathrm{bA}$ & $0,011 \mathrm{bA}$ \\
\hline TSKC $\times($ LCR $\times$ TR $)-040$ & $0,018 \mathrm{a}$ & $0,024 \mathrm{aA}$ & $0,027 \mathrm{aA}$ & $0,028 \mathrm{aA}$ & $0,028 \mathrm{aA}$ \\
\hline HTR - 116 & $0,011 \mathrm{a}$ & $0,011 \mathrm{bA}$ & $0,007 \mathrm{bA}$ & $0,010 \mathrm{cA}$ & $0,007 \mathrm{bA}$ \\
\hline HTR - 069 & $0,020 \mathrm{a}$ & $0,030 \mathrm{aA}$ & $0,027 \mathrm{aA}$ & $0,029 \mathrm{aA}$ & $0,026 \mathrm{aA}$ \\
\hline
\end{tabular}

Letras maiúsculas distintas indicam diferença significativa entre híbridos pelo teste de Skott-Knott ao nível de 0,05 de probabilidade e letras minúsculas distintas indicam diferença significativa para os níveis de salinidade pelo teste de Tukey a 0,05 de probabilidade.

na verificação do início da percepção do estresse e ocorrência de mecanismos de tolerância.

Por ser responsável pelo controle das trocas gasosas, o fechamento dos estômatos, observado às $24 \mathrm{~h}$ no HTR-127 e às 48 horas no HTR-069, determinou reduções na taxa de assimilação de $\mathrm{CO}_{2}$ desses híbridos, o que pode estar relacionado ao menor influxo de $\mathrm{CO}_{2}$ na cavidade subestomática para realização do fenômeno da fotossíntese (Taiz \& Zaiger, 2009), já que após a estabilização da condutância estomática constatou-se no HTR-127, sob condições de salinidade, uma ampliação da atividade fotossintética em relação ao que se deu em plantas desse genótipo no menor nível de salinidade da água, fato que pode estar relacionado a uma maior necessidade de solutos para regularização dos efeitos ocasionados pelo estresse salino (Taiz \& Zaiger, 2009). 
Ao se estudar a eficiência do uso da água em híbridos de citros sob condições de salinidade da água (Tabela 3), nota-se que o LCR x TR - 001 sofreu reduções nessa variável quando submetido ao maior nível de salinidade $\left(4,0 \mathrm{dS} \mathrm{m}^{-1}\right)$ durante as primeiras $24 \mathrm{~h}$, mantendo-se constante até a avaliação às 48 horas. Efeito contrário foi observado no híbrido TSKC x (LCR x TR) - 040, em relação ao qual se verificou aumento da eficiência do uso da água durante todo período de avaliação. Tal observação permite deduzir que esse genótipo possui rápida percepção do estresse salino, assim como tolerância, já que manteve a EUA nas avaliações às 24 e $48 \mathrm{~h}$ após início da submissão ao estresse (Tabela 3).

A eficiência no uso da água, expressa pela relação entre a fotossíntese e a transpiração, está relacionada à quantidade de carbono que a planta fixa por cada unidade de água que perde (Taiz \& Zeiger, 2009). Dessa forma, plantas que tenham capacidade de aumentar a eficiência no uso da água sob condições de salinidade, como o observado no híbrido TSKC x (LCR x TR) - 040, possivelmente apresentem alta capacidade de tolerância ao estresse salino, já que a redução no consumo de água implica em redução na absorção de íons específicos, evitando, portanto, efeitos tóxicos na planta (Flowers \& Flowers, 2005), o que pode estar relacionado ao mecanismo de exclusão de sais pelas raízes (Taiz \& Zeiger, 2009).

Ainda, uma menor eficiência no uso da água, sob condições de salinidade, pode indicar comprometimento da atividade fisiológica, fato evidenciado no LCR x TR - 001 que, além de redução nessa variável, apresentou redução da eficiência quântica do fotossitema II (Fv/Fm) (Tabela 5).

Com respeito à eficiência instantânea da carboxilação, apenas o HTR-127 manifestou diferenças significativas em resposta aos níveis de salinidade da solução, observando-se reduções nas primeiras $24 \mathrm{e} 48 \mathrm{~h}$ de aplicação dos tratamentos. Tais reduções estão relacionadas a diminuições, tanto na condutância estomática quanto da taxa de assimilação de $\mathrm{CO}_{2}$, em consequência do estresse salino, que pode ter estabilizado a atividade estomática e a fotossíntese líquida (Tabela 3). Todavia, cabe indicar a possibilidade de algum fator não-estomático ter interferido na eficiência instantânea da carboxilação, o que poderá ser confirmado quando se analisar a fluorescência da clorofila $a$.

No estudo de fluorescência da clorofila $a$, observou-se que os híbridos avaliados divergiram entre si, mesmo na ausência da aplicação de solução salina (0 hora), quanto à fluorescência inicial (Fo), fluorescência máxima (Fm) e fluorescência variável (Fv) (Tabela 4). Na avaliação às $24 \mathrm{~h}$, constatou-se diferenças entre os híbridos quanto à Fo e à relação Fv/Fm, nesta variável, ainda, verificou-se diferenças entre as soluções salinas. Às 24 horas foram observadas, também, interações significativas entre os fatores salinidade da água e híbridos para as fluorescências máxima e variável. Na avaliação às 48 h, destacou-se a ocorrência de diferenças entre os híbridos em todas as variáveis analisadas, e da salinidade na solução na Fm, Fv e relação $\mathrm{Fv} / \mathrm{Fm}$, ademais, o efeito da interação perdurou apenas na Fm e na Fv (Tabela 4).
Tabela 4. Resumo da análise de variância das variáveis de fluorescência da clorofila a: fluorescência inicial (Fo), fluorescência máxima $(\mathrm{Fm})$, fluorescência variável (Fm-Fo) e eficiência quântica do fotossistema II (Fv/ Fm) de híbridos de citros submetidos à salinidade da água em cultivo hidropônico, considerando diferentes tempos após o início da aplicação do estresse: 0, 24 e 48 horas

\begin{tabular}{|c|c|c|c|c|c|}
\hline \multirow{2}{*}{$\begin{array}{c}\text { Fontes de } \\
\text { variação }\end{array}$} & \multirow{2}{*}{ GL } & \multicolumn{4}{|c|}{ Quadrado médio } \\
\hline & & Fo Oh & Fm Oh & Fv Oh & Fv/Fm Oh \\
\hline Híbridos & 3 & $4.347,56$ ** & $68.723,10^{\star *}$ & $55.083,06$ ** & $0,00055^{\mathrm{NS}}$ \\
\hline Blocos & 2 & $192,46^{\mathrm{NS}}$ & $410,60^{\mathrm{NS}}$ & $2.094,86^{\mathrm{NS}}$ & $0,00004^{\mathrm{NS}}$ \\
\hline Erro & 6 & 309,96 & $7.271,85$ & $6.084,11$ & 0,00017 \\
\hline \multirow[t]{2}{*}{ CV } & & 4,52 & 3,86 & 4,28 & 1,60 \\
\hline & & Fo 24h & $\mathrm{Fm} \mathrm{24h}$ & Fv 24h & Fv/Fm 24h \\
\hline Salinidade & 1 & $30.464,53^{*}$ & $22,53^{\mathrm{NS}}$ & $28.830,00^{N S}$ & $0,0078 * \star$ \\
\hline Bíbridos & 4 & $10.493,38^{N S}$ & $66.771,20$ ** & $78.372,53^{\star *}$ & $0,0036^{\star *}$ \\
\hline $\begin{array}{l}\text { Salinidade x } \\
\text { Híbridos }\end{array}$ & 4 & $6.786,28^{\mathrm{NS}}$ & $110.280,53^{\star *}$ & $88.984,66^{\star *}$ & $0,0015^{\text {NS }}$ \\
\hline Blocos & 2 & $5.528,03^{\text {NS }}$ & $27.736,43^{\mathrm{NS}}$ & $10.213,23^{\text {NS }}$ & $0,0003^{\mathrm{NS}}$ \\
\hline Erro & 18 & $3.936,81$ & $10.460,72$ & $7.590,12$ & 0,0007 \\
\hline \multirow[t]{2}{*}{ CV } & & 13,61 & 5,08 & 5,61 & 3,03 \\
\hline & & Fo 48h & Fm 48h & Fv 48h & $\mathrm{Fv} / \mathrm{Fm} \mathrm{48h}$ \\
\hline Salinidade & 1 & $2.430,00^{\mathrm{NS}}$ & $55.298,13^{*}$ & $80.912,13^{* *}$ & $0,00217^{\star}$ \\
\hline Híbridos & 4 & $22.305,66^{\star \star}$ & $282.108,96$ ** & $201.714,83^{* *}$ & 0,00266 ** \\
\hline $\begin{array}{l}\text { Salinidade x } \\
\text { Híbridos }\end{array}$ & 4 & $4.964,00^{\mathrm{NS}}$ & $177.413,63^{* *}$ & $138.329,13^{* *}$ & $0,00056^{\mathrm{NS}}$ \\
\hline Blocos & 2 & $2.298,53$ & $83.328,70$ ** & $60.051,60$ ** & $0,00011^{\mathrm{NS}}$ \\
\hline erro & 18 & $3.005,27$ & $11.132,07$ & $8.126,71$ & 0,00045 \\
\hline CV & & 12,42 & 4,96 & 5,35 & 2,69 \\
\hline
\end{tabular}

${ }^{*},{ }^{\star *}=$ significante a nível de 0,05 e 0,01 de probabilidade; ${ }^{N S}=$ não significativo; $\mathrm{GL}=$ grau de liberdade; $C V=$ coeficiente de variação.

Os resultados de fluorescência da clorofila $a$ apresentaram-se em consonância com os observados para trocas gasosas (Tabela 2), reforçando os postulados de Ayers \& Westcot (1999), de que as repostas aos efeitos da salinidade variam entre as espécies e entre plantas de uma mesma espécie. Semelhantemente, Cruz et al. (2009), estudando combinações copa-porta-enxerto de citros sob estresse hídrico, verificaram efeito significativo nas variáveis de fluorescência, relacionando essa resposta a danos no fotossistema.

O maior nível de salinidade utilizado $\left(4,0 \mathrm{dS} \mathrm{m}^{-1}\right)$ aumentou a fluorescência inicial nos híbridos citros, em relação ao que se verificou no menor nível $\left(0,3 \mathrm{dS} \mathrm{m}^{-1}\right)$ (Tabela 5), sendo o maior incremento observado no HTR-127, da ordem de 155 fotons. A fluorescência inicial explica a fluorescência quando a quinona, receptora primária de elétrons (QA) do fotossistema II (PSII), está totalmente oxidada e o centro de reação (P680) está aberto, indicando ativação das reações fotoquímicas (Munns \& Tester, 2008; Lucena et al., 2012). Pode-se concluir que a salinidade afetou a atividade do fotossitema II dos híbridos nas primeiras 24 horas de submissão à salinidade, indicando o início da percepção do estresse a partir desse momento para todos os genótipos de citros estudados (Tabela 5), o que permite dizer que esta é uma das variáveis recomendadas para avaliação do início do estresse. Ainda, tal estresse foi mais evidente no HTR-127, em relação ao qual ocorreram diferenças significativas quanto à eficiência instantânea da carboxilação, o que vem a confirmar a influência de fatores não-estomáticos na redução da fotossíntese verificada nesse indivíduo. 
Quando estudada a fluorescência máxima da clorofila, foram verificados efeitos da salinidade sob os híbridos HTR-127 e HTR-069 durante as 48 horas de submissão à salinidade (Tabela 5). Para o HTR-127 observou-se uma ampliação na fluorescência máxima, o que pode estar relacionado a uma compensação do processo fotossintético em busca de aumentar a taxa de assimilação de $\mathrm{CO}_{2}$, já que esta foi drasticamente reduzida nas primeiras 24 horas e se estabilizou após as 48 horas de aplicação dos tratamentos.

No HTR-069 verificou-se um efeito contrário, pois a fluorescência máxima deste híbrido decresceu durante as 48 horas de aplicação dos tratamentos quando o mesmo foi submetido ao maior nível de salinidade $\left(4,0 \mathrm{dS} \mathrm{m}^{-1}\right)$. Essas reduções vão ao encontro das reduções na taxa de assimilação de $\mathrm{CO}_{2}$, o que indica uma desaceleração na atividade fotossintética visando minimizar os efeitos tóxicos da salinidade, de modo a diminuir o fluxo de entrada de água na planta e, com isso, evitar a toxicidade por excesso de íons específicos dentro da planta (Flowers \& Flowers, 2005), facilitando a exclusão do sal pelo sistema radicular (Taiz \& Zeiger, 2009).

Quanto à fluorescência variável, apenas o híbrido TSKC $\mathrm{x}$ (LCR x TR) - 040 não sofreu alterações quando submetido ao tratamento salino, o que pode estar relacionado à sua maior

Tabela 5. Teste de médias das variáveis de fluorescência da clorofila a: fluorescência inicial (Fo), fluorescência máxima (Fm), fluorescência variável (Fv) e eficiência quântica do fotossistema II (Fv/Fm) de híbridos de citros submetidos à salinidade da água em cultivo hidropônico, considerando diferentes tempos após o início da aplicação do estresse: 0, 24 e 48 horas

\begin{tabular}{|c|c|c|c|c|c|}
\hline \multirow{3}{*}{ Híbridos } & \multirow{2}{*}{$\begin{array}{c}0 \text { hora } \\
0,3\end{array}$} & \multicolumn{2}{|c|}{24 horas } & \multicolumn{2}{|c|}{48 horas } \\
\hline & & 0,3 & 4,0 & 0,3 & 4,0 \\
\hline & \multicolumn{5}{|c|}{$\mathrm{dSm}^{-1}$} \\
\hline \multicolumn{6}{|c|}{ Fluorescência inicial (Fo) } \\
\hline HTR - 127 & $443 a$ & $395 \mathrm{aB}$ & $550 \mathrm{aA}$ & $307 \mathrm{cA}$ & $400 \mathrm{cA}$ \\
\hline LCR x TR - 001 & $368 b$ & $416 \mathrm{aB}$ & $497 \mathrm{aA}$ & $433 \mathrm{bA}$ & $482 \mathrm{bA}$ \\
\hline TSKC $\times($ LCR $\times$ TR $)-040$ & $358 b$ & $407 a B$ & $390 a A$ & $420 \mathrm{bA}$ & $440 \mathrm{bA}$ \\
\hline HTR - 116 & $362 b$ & $508 \mathrm{aB}$ & $522 \mathrm{aA}$ & $551 a A$ & $496 \mathrm{aA}$ \\
\hline HTR - 069 & $417 \mathrm{a}$ & $419 \mathrm{aB}$ & $504 \mathrm{aA}$ & $450 \mathrm{bA}$ & $432 \mathrm{bA}$ \\
\hline \multicolumn{6}{|c|}{ Fluorescência máxima (Fm) } \\
\hline HTR - 127 & $2259 a$ & $1967 \mathrm{bB}$ & $2370 \mathrm{aA}$ & $1547 \mathrm{bB}$ & $2006 \mathrm{bA}$ \\
\hline LCR x TR - 001 & $2267 a$ & $2005 \mathrm{bA}$ & $2048 \mathrm{bA}$ & $2481 b A$ & $2209 a A$ \\
\hline TSKC $\times($ LCR $\times$ TR $)-040$ & 1940b & $1986 \mathrm{bA}$ & $1899 \mathrm{bA}$ & $2241 \mathrm{bA}$ & $2275 \mathrm{aA}$ \\
\hline HTR - 116 & $2296 a$ & $1893 \mathrm{bA}$ & $1891 \mathrm{bA}$ & $2268 \mathrm{bA}$ & $2020 \mathrm{bA}$ \\
\hline HTR - 069 & $2286 a$ & $2210 \mathrm{aA}$ & $1860 \mathrm{bB}$ & $2309 a A$ & $1906 \mathrm{bB}$ \\
\hline \multicolumn{6}{|c|}{ Fluorescência variável (Fv) } \\
\hline HTR - 127 & $1832 \mathrm{a}$ & $1572 \mathrm{bA}$ & $1820 \mathrm{aB}$ & $1240 c B$ & $1606 \mathrm{bA}$ \\
\hline LCR x TR - 001 & $1898 a$ & $1589 \mathrm{bA}$ & $1551 b B$ & $2048 a A$ & $1726 \mathrm{aB}$ \\
\hline TSKC $\times($ LCR $x$ TR $)-040$ & $1585 b$ & $1579 \mathrm{bA}$ & $1509 b A$ & $1821 \mathrm{bA}$ & $1835 \mathrm{aA}$ \\
\hline HTR - 116 & $1918 \mathrm{a}$ & $1385 \mathrm{cA}$ & $1369 \mathrm{cA}$ & $1717 \mathrm{bA}$ & $1523 b B$ \\
\hline HTR - 069 & $1869 a$ & $1790 \mathrm{aA}$ & $1356 \mathrm{cB}$ & $1858 \mathrm{bA}$ & $1474 \mathrm{bB}$ \\
\hline \multicolumn{6}{|c|}{ Eficiência quântica do fotossistema II (Fv/Fm) } \\
\hline HTR - 127 & $0,80 \mathrm{a}$ & $0,80 \mathrm{aA}$ & $0,77 \mathrm{aA}$ & $0,80 \mathrm{aA}$ & $0,80 \mathrm{aA}$ \\
\hline LCR x TR - 001 & $0,83 a$ & $0,79 \mathrm{aA}$ & $0,76 \mathrm{aA}$ & $0,83 \mathrm{aA}$ & $0,78 \mathrm{aB}$ \\
\hline TSKC $\times($ LCR $\times$ TR $)-040$ & $0,81 \mathrm{a}$ & $0,79 \mathrm{aA}$ & $0,79 \mathrm{aA}$ & $0,81 \mathrm{aA}$ & $0,81 a A$ \\
\hline HTR - 116 & $0,83 a$ & $0,74 \mathrm{bA}$ & $0,72 \mathrm{bB}$ & $0,76 \mathrm{bA}$ & $0,75 a A$ \\
\hline HTR - 069 & $0,81 a$ & $0,81 \mathrm{aA}$ & $0,73 \mathrm{bB}$ & $0,80 \mathrm{aA}$ & $0,77 a A$ \\
\hline
\end{tabular}

Letras maiúsculas distintas indicam diferenças significativas entre híbridos pelo teste de Skott-Knott ao nível de 0,05 de probabilidade e letras minúsculas diferentes indicam diferença significativa para os níveis de salinidade pelo teste de Tukey ao nível de 0,05 de probabilidade. tolerância aos efeitos da salinidade, já que não se verificou diferenças nas trocas gasosas desse genótipo (Tabelas 3 e 5).

Ainda sobre a Fv, foi verificado que a salinidade reduziu a expressão desta variável nos híbridos trifoliados LCR x TR - 001 e HTR-069 às 24 e 48 horas, sendo que em relação ao HTR-116 ocorreu redução somente às 48 horas (Tabela 5). No entanto para o híbrido HTR-127 a fluorescência variável oscilou durante a aplicação dos tratamentos, apresentando redução nas primeiras 24 h e aumento de sua expressão após as 48 horas, observando-se o mesmo em relação à taxa de assimilação de $\mathrm{CO}_{2}$, indicando uma estabilização do efeito do estresse nesse indivíduo (Tabelas 3 e 5).

Apenas no híbrido LCR x TR - 001 foram constatadas reduções significativas na eficiência quântica do fotossistema II, com valores indo de 0,83 no nível de $0,3 \mathrm{dS} \mathrm{m}^{-1}$ para 0,78 no nível

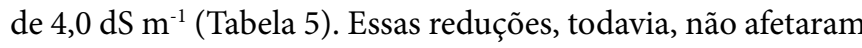
as trocas gasosas desse híbrido, pois o valor inferior observado $(0,78)$ mostrou-se superior ao limite para comprometimento do fotossistema $(0,75)$. Mendonça et al. (2010) também observaram reduções na eficiência quântica do fotossistema II em diferentes espécies de eucalipto sob salinidade da água de irrigação.

\section{Conclusões}

1. As variáveis fluorescência inicial, condutância estomática e eficiência no uso da água podem ser usadas para descrever a percepção do estresse salino nos híbridos estudados.

2. A salinidade reduziu a atividade do fotossistema II do híbrido LCR x TR - 001, sendo este o genótipo mais sensível à salinidade dentre os indivíduos estudados.

3. O híbrido trifoliado HTR-127 foi o primeiro a estabilizar a atividade fotossintética após a percepção do estresse salino.

4. O híbrido HTR-116 sofreu efeito da salinidade apenas na fluorescência da clorofila $a$.

5. O HTR-069 reduziu sua atividade fotossintética de modo a minimizar os efeitos da salinidade, destacando-se como o híbrido que apresentou os melhores valores de trocas gasosas.

6. A eficiência no uso da água pelo híbrido TSKC x (LCR x TR) - 040 foi potencializada sob condições de salinidade, distinguindo-se este genótipo por sua maior capacidade de tolerância ao estresse salino, sendo seguido pelo HTR-069.

\section{Agradecimentos}

Ao CNPq, pela manutenção das bolsas PIBITI e Produtividade em Pesquisa e o financiamento por meio do Edital Universal. À Embrapa Mandioca e Fruticultura, pela parceria. Aos colegas da equipe de trabalho, pelo empenho e dedicação.

\section{Literatura Citada}

Agrianual. Anuário da agricultura brasileira. São Paulo: FNP Consultório e Comércio, 2010. 520p.

Ayers, R. S.; Westcot, D. W. Qualidade da água na agricultura. Campina Grande: UFPB. 1999. 153p. Estudos FAO: Irrigação e Drenagem, 29 
Brito, M. E. B.; Fernandes, P. D.; Gheyi, H. R.; Melo, A. S. de; Cardoso, J. A. F.; Soares Filho, W. S. Sensibilidade de variedades e híbridos de citrange à salinidade na formação de porta-enxertos. Revista Brasileira de Ciências Agrárias, v.3, p.343-353, 2008.

Brito, M. E. B.; Soares, L. A. dos A.; Fernandes, P. D.; Lima, G. S.; Sá, F. V. da S.; Melo, A. S. Comportamento fisiológico de combinações copa/porta-enxerto de citros sob estresse hídrico. Revista Brasileira de Ciências Agrárias, v.7, suppl., p.857-865, 2012.

Cruz, M. C. M. da; Siqueira, D. L. de; Salomão, L. C. C.; Cecon, P. R. Fluorescência da clorofila $a$ em folhas de tangerineira 'Ponkan' e limeira ácida 'Tahiti' submetidas ao estresse hídrico. Revista Brasileira de Fruticultura, v.31, p.896-901, 2009.

Fernandes, P. D.; Brito, M. E. B.; Gheyi, H. R.; Soares Filho, W. dos S.; Melo, A. S. de; Carneiro, P. T. Crescimento de híbridos e variedades porta-enxerto de citros sob salinidade. Acta Scientiarum. Agronomy, v.33, p.259-267, 2011.

Ferreira, D. F. Análises estatísticas por meio do Sisvar para Windows versão 4.0. In: Reunião Anual da Região Brasileira da Sociedade Internacional de Biometria, 2000, São Carlos. Anais... São Carlos: UFSCar. 2000. p.255-258.

Flowers, T. J.; Flowers, S. A. Why does salinity pose such a difficult problem for plant breeders? Agricultural Water Management, v.78, p.15-24, 2005.

Hoagland, D. R.; Arnon, D. I. The water-culture method for growing plants without soil.Berkeley, California Agricultural Experiment Station, 1950. 32p. Circular, 347

Hussain, S.; Luro, F.; Costantino, G.; Ollitrault, P.; Morillon, R. Physiological analysis of salt stress behavior of citrus species and genera: Low chloride accumulation as an indicator of salt tolerance. South African Journal of Botany, v.81, p.103112, 2012.

Jamil, M.; Rehman, S.; Lee, K. J.; Kim, J. M.; Kim, H.; Rha, E. S. Salinity reduced growth PS2 photochemistry and chlorophyll content in radish. Scientia Agricola, v.64, p.111118, 2007.

Konrad, M. L. F.; Silva, J. A. B.; Furlani, P. R.; Machado, E. C. Trocas gasosas e fluorescência da clorofila em seis cultivares de cafeeiro sob estresse de alumínio. Bragantia, v.64, p.339$347,2005$.
Lucena, C. C.; Siqueira, D. L.; Martinez, H. E. P.; Cecon, P. R. Salt stress change chlorophyll fluorescence in mango. Revista Brasileira Fruticultura, v.34, p.1245-1255, 2012.

Machado, E. C.; Schmidt, P. T.; Medina, C. L.; Ribeiro, R. V. Respostas da fotossíntese de três espécies de citros a fatores ambientais. Pesquisa Agropecuária Brasileira, v.40, p.11611170, 2005.

Magalhães Filho, J. R.; Amaral, L. R.; Machado, D. F. S. P.; Medina, C. L.; Machado, E. C. Deficiência hídrica, trocas gasosas e crescimento de raízes em laranjeira 'valência' sobre dois tipos de porta-enxerto. Bragantia, v.67, p.75-82, 2008.

Mattos Júnior, D.; Negri, J. D. de; Pio, R. S.; Pompeu Júnior, J. Citros. Campinas: Instituto Agronômico e FUNDAG, 2005. 929p.

Maxwell, K.; Johnson, G. N. Chlorophyll fluorescence: a practical guide. Journal Experimental Botany, v.51, p.659-668, 2000.

Medeiros, J. F. de; Lisboa, R. de A.; Oliveira, M. de; Silva Júnior, M. J. da; Alves, L. P. Caracterização das águas subterrâneas usadas para irrigação na área produtora de melão da Chapada do Apodi. Revista Brasileira Engenharia Agrícola e Ambiental, v.7, p.469-472, 2003.

Mendonça, A. V. R.; Carneiro, J. G. A.; Freitas, T. A. S.; Barroso, D. G. Características fisiológicas de mudas de Eucalyptus spp. submetidas a estresse salino. Ciência Florestal, v.20, p.255-267, 2010.

Munns, R.; Tester, M. Mechanisms of salinity tolerance. Annual Reviews of Plant Biology, v.59, p.651-681, 2008.

Nascimento, A. K. S.; Fernandes, P. D.; Suassuna, J. F.; Oliveira, A. C. M.; Sousa, M. S. S.; Azevedo, J. G. N. Tolerância de genótipos de citros ao estresse hídrico na fase de portaenxerto. Irriga, Edição Especial, p.438-452, 2012.

Santos, C. E. R. S; Bezerra, R. V.; Freitas, A. D. S.; Seido, S. L.; Martins, L. M. V.; Rumjanek, N. G.; Xavier, G. R. Modificação de vasos de Leonard com garrafas descartáveis tipo Pet. Seropédica: Embrapa, 2009. 4p. Comunicado Técnico 124

Silva, F. V. da; Soares, F. A. L.; Gheyi, H. R.; Travassos, K. D.; Suassuna, J. F.; Cardoso, J. A. F. Produção de citros irrigados com água moderadamente salina. Irriga, Edição Especial, p.396-407, 2012.

Singh, A.; Saini, M. L.; Behl, R. K. Screening of citrus rootstocks for salt tolerance in semi-arid climates - A review. Tropics, v.13, p.53-66, 2003.

Taiz, L.; Zeiger, E. Fisiologia vegetal. 4.ed. Porto Alegre: Artmed, 2009. 819p. 